\title{
Editorial
}

Nephrology

\section{Failure in Physiologic Flexibility: Adverse Pregnancy Outcomes in Women with Reduced Renal Reserve}

\author{
Elizabeth Lemoine ${ }^{\mathrm{a}, \mathrm{b}} \quad$ S. Ananth Karumanchi ${ }^{\mathrm{a}-\mathrm{c}}$ \\ ${ }^{a}$ Department of Medicine, Cedars-Sinai Medical Center, Los Angeles, CA, USA; ${ }^{b}$ Harvard Medical School, Boston, MA, \\ USA; ${ }^{C}$ Center for Vascular Biology Research, Beth Israel Deaconess Medical Center, Boston, MA, USA
}

A healthy pregnancy undergoes significant physiologic changes requiring multiorgan plasticity. Failure to adapt to the hemodynamic and humoral changes of gestation due to acute or chronic disease puts mother and fetus at risk for potentially life-threatening complications. Responding to an increase in nitric oxide and relaxin and a decrease in sensitivity to vasoconstrictors, the renal vasculature adapts in normal pregnancy to accommodate the large increase in blood volume and renal blood flow. Pre-pregnancy kidney disease that limits this remodeling increases the risk for complications, most notably pregnancy-induced hypertension [1-3].

Preeclampsia-eclampsia, a hypertensive disorder of pregnancy and one of the most globally fatal complications, results from placental overproduction of soluble fms-like tyrosine kinase, causing systemic endothelial damage, hypertension, and end-organ failure [4]; the prepregnancy endothelial damage and vasculopathy of diabetes mellitus, obesity, or chronic hypertension increase a woman's baseline risk of developing preeclampsia by decreasing her renal and vascular reserve. In addition to baseline systemic endothelial damage, women with chronic kidney disease (CKD) lack the glomerular plasticity to respond to pregnancy-induced hormonal chang-

\section{KARGER}

(c) 2019 S. Karger AG, Basel

E-Mail karger@karger.com

www.karger.com/ajn es and fail to hyperfiltrate sufficiently to accommodate the increased volume of pregnancy. The relative hypofiltration prevents adequate volume expansion leading to placenta ischemia and an increased risk of preeclampsiaeclampsia. In order to optimize prenatal care and appropriate referral, it is important to identify the pregnancy risks associated with pre-pregnancy comorbidities limiting maternal physiologic flexibility.

Although efforts have been made to determine the effects of renal disease overall on pregnancy outcomes, data on which adverse pregnancy outcomes correlate with individual renal pathologies are limited. It is logical that a vascular renal disease would put a woman at higher risk for a vascular complication of pregnancy such as preeclampsia; but is this what is observed at the population level? In "The Spectrum of Adverse Pregnancy Outcomes based on Kidney Disease Diagnoses: a 20 -year population study" published in this issue, a South Australian group led by Fitzpatrick et al. [5] identifies the prevalence of pregnancy-related outcomes among kidney disorders in the 407,580 births recorded in the South Australian Pregnancy Outcomes Unit (1990-2012). As the authors note, strengths of the study include the large population and the low systematic bias due to the mandatory reporting 
that generated the database. However, the study is limited by diagnoses captured by ICD-9 codes without an ability to confirm diagnoses with laboratory, imaging, or pathology data, subjecting them to reporting bias and an inability to validate diagnoses. Furthermore, data collection is mandated only for births $>20$ weeks gestation, suggesting that women with the greatest systemic illnesses and therefore with the greatest number of spontaneous abortions such as antiphospholipid syndrome/systemic lupus erythematous - may have been excluded from analysis.

Overall, the study confirms findings of previous literature that women with renal disease are at an increased risk of pregnancy-induced hypertension, cesarean section (an illness severity marker), low birth weight, preterm delivery, and NICU admission [3]. However, the large size of the cohort allows for sub-analyses of diseases grouped by pathologies: immunological or cystic/genetic conditions, vesicoureteral reflux (VUR), urological conditions, pyelonephritis and "other" including renal disease secondary to diabetes mellitus and "unspecified renal disease in pregnancy." Perhaps the most interesting aspect in this subgroup analysis is the fact that all adverse pregnancy outcomes appear to be driven by an increased risk associated with immunological disease and/or VUR. Though large, prospective studies observe that advancing stage of CKD increases the risk of gestational complications [3], Fitzpatrick et al. [5] further suggest that particular etiologies of acute and chronic renal disease, not just disease progression, may drive adverse pregnancy outcomes.

Previous meta-analyses of VUR and associated adverse pregnancy outcomes suggest a considerably more benign scenario. Fitzpatrick et al. [5] large cohort confirms the previous meta-analyses' observations of an increased risk of pregnancy-induced hypertension and preeclampsia [6, 7]; however, one meta-analysis could only identify a risk of hypertension in the presence of renal scarring [7], emphasizing the importance of disease progression on outcomes. Fitzpatrick et al. [5] suggest in their findings a much graver pathology, observing an increased risk of preterm delivery, low birth weight, NICU admission, and cesarean delivery. Due to limitations in the database, the percentage of renal scarring and severity of renal dysfunction (i.e., degree of proteinuria or creatinine elevation) cannot be deduced. Severe baseline scarring in this population would prevent renal physiologic adaption to pregnancy, resulting in systemic markers of poor pregnancy health. We speculate that biochemical validation of the degree of disease progression would have demonstrated a more advanced stage of renal disease in this VUR population.
While Fitzpatrick et al. [5] contribute to a bird's eye view of kidney disease in pregnancy, the risk of poor pregnancy outcomes - particularly with regard to hypertensive disorders of pregnancy - has been characterized within certain individual renal diseases. Recent data suggest that acute, recovered disease - not just chronic disease - affects the health of a pregnancy; Tangren et al. [8] demonstrate that a history of clinically recovered acute kidney injury significantly increases a woman's risk for preeclampsia and small for gestational age infants, as well as her rate of cesarean delivery and NICU admission. Interestingly, the proximity of the AKI to conception (within 18 months) increased the risk of preeclampsia sevenfold, re-enforcing the importance of current maternal renal reserve in maintaining a healthy pregnancy.

$\mathrm{CKD}$ is a known risk-factor for complications in pregnancy $[1,3]$ due to an inability to accommodate adequate volume expansion. About $30 \%$ of women with pre-existing chronic hypertension will develop superimposed preeclampsia [4] likely due to placental ischemia from inadequate supporting volume. Furthermore, chronic systemic disease with renal involvement - such as systemic lupus erythematous and diabetes mellitus - has a welldocumented risk of developing hypertensive disorders of pregnancy [4]. Studies including populations with single kidneys reinforce the association between decreased renal reserve and hypertensive disorders of pregnancy. Women with single functioning kidneys due to congenital renal agenesis or transplant recipiency have increased preterm preeclampsia, and low birth weight $[6,9]$, while women status post kidney donation have excess risk for term preeclampsia [10]. The comparison reinforces that duration or severity of renal disease at the time of conception leads to systemic consequences and therefore inferior fetal outcomes.

Associative, population-level analyses like those of Fitzpatrick et al. [5] cannot provide causation, but they do offer a starting place for inquisition. Future studies with animal models of VUR are needed for better understanding of the high risk of pregnancy complications the researchers observed in this population. However, Fitzpatrick et al. [5] bring more greatly needed attention to how a woman's pre-pregnancy health affects her pregnancy, her neonate, and her long-term health risks. Though Fitzpatrick et al. [5] analysis only included kidney disease concurrent with pregnancy and not its history, Tangren et al. [8] findings suggest that a history of recovered renal disease may provide insight into a woman's baseline renal function and therefore her risk for preeclampsia. 
A history of preeclampsia is a known risk factor for cardiovascular disease, increasing a woman's risk for fatal myocardial infarction, acute coronary syndrome, vascular dementia, stroke, and end-stage renal disease [4]. Identifying women with decreased renal reserve prior to conception and optimizing their care may reduce their long-term risk of cardiovascular complications or worsening of preexisting disease. Fitzpatrick et al. [5] assess- ment of the association of kidney disorder diagnoses with adverse pregnancy outcomes reminds us that pregnancy is a demanding physiological state with the unique quality of a prenatal window of intervention prior to initiation of the stress. Addressing preexisting health issues prenatally not only maximizes pregnancy outcomes for mother and child but may serve the mother's health for many post-partum years to come.

\section{References}

1 Hladunewich MA. Chronic Kidney Disease and Pregnancy. Semin Nephrol. 2017 Jul; 37(4):337-46.

2 Piccoli GB, Attini R, Vasario E, Conijn A, Biolcati M, D'Amico F, et al. Pregnancy and chronic kidney disease: a challenge in all CKD stages. Clin J Am Soc Nephrol. 2010 May;5(5): $844-55$

3 Piccoli GB, Cabiddu G, Attini R, Vigotti FN, Maxia S, Lepori N, et al. Risk of Adverse Pregnancy Outcomes in Women with CKD. J Am Soc Nephrol. 2015 Aug;26(8):2011-22.

4 Phipps EA, Thadhani R, Benzing T, Karumanchi SA. Pre-eclampsia: pathogenesis, novel diagnostics and therapies. Nat Rev Nephrol. 2019 Feb. https://doi.org/10.1038/ s41581-019-0119-6.
5 Fitzpatrick A, Venugopal K, Scheil W, McDonald SP, Jesudason S. The Spectrum of Adverse Pregnancy Outcomes based on Kidney Disease Diagnoses: a 20-year population study. Am J Nephrol. 2019;49:400-9.

6 Attini R, Kooij I, Montersino B, Fassio F, Gerbino M, Biolcati M, et al. Reflux nephropathy and the risk of preeclampsia and of other adverse pregnancy-related outcomes: a systematic review and meta-analysis of case series and reports in the new millennium. J Nephrol. 2018 Dec;31(6):833-46.

7 Hollowell JG. Outcome of pregnancy in women with a history of vesico-ureteric reflux. BJU Int. 2008 Sep;102(7):780-4.
8 Tangren JS, Wan Md Adnan WA, Powe CE, Ecker J, Bramham K, Hladunewich MA, et al. Risk of Preeclampsia and Pregnancy Complications in Women With a History of Acute Kidney Injury. Hypertension. 2018 Aug; 72(2):451-9.

9 Mohammadi FA, Borg M, Gulyani A, McDonald SP, Jesudason S. Pregnancy outcomes and impact of pregnancy on graft function in women after kidney transplantation. Clin Transplant. 2017 Oct;31(10):31.

10 Garg AX, Nevis IF, McArthur E, Sontrop JM Koval JJ, Lam NN, et al.; DONOR Network. Gestational hypertension and preeclampsia in living kidney donors. N Engl J Med. 2015 Jan;372(2):124-33. 